\title{
Preemptive Capacity Investment under Uncertainty
}

\author{
Benoît Chevalier-Roignant ${ }^{\mathrm{a}, *}$, Lenos Trigeorgis ${ }^{\mathrm{b}}$ \\ ${ }^{a}$ WHU-Otto Beisheim School of Management, \\ Burgplatz 2, 56179 Vallendar, Germany \\ ${ }^{b}$ University of Cyprus, Department of Public and Business Administration, \\ 9-11 Larnakas Avenue, 1678 Nicosia, Cyprus
}

\begin{abstract}
The incentive to "overinvest" in capital may be eroded in dynamic, competitive settings if firms face uncertainty and irreversibility. In this paper, we derive the stationary Markov perfect equilibrium for a dynamic, infinite-horizon capacity investment game formulated in continuous time in which reduced-form profits are subject to industry shocks. We show that the theory of marginal Tobin's $q$ still holds in capital-accumulation games under uncertainty if the strategic externalities of rivals' investment are properly accounted for.

Keywords: financial economics, strategic investment under uncertainty, real options, stochastic differential games, capital accumulation
\end{abstract}

\section{Introduction}

It is standard practice in finance and strategy to interpret real investment opportunities as being analogous to options. This view is well accepted among academics and practitioners alike and is the core of real options analysis (ROA), as summarized in the works of Dixit and Pindyck (1994) and Trigeorgis (1996). Since standard ROA draws from contingent-claims analysis based on financial options, a key feature specific to real assets is often ignored - namely, the interplay or strategic interactions taking place among (real) option holders. Bridging this gap requires the use of additional notions borrowed from (dynamic) game theory. Chevalier-Roignant and Trigeorgis (2011) develop further the notion of "option games," while Chevalier-Roignant et al. (2010) review the relevant literature.

\footnotetext{
* Corresponding author

Email addresses: benoit.chevalier-roignant@whu.edu (Benoît Chevalier-Roignant), lenos@ucy.ac.cy (Lenos Trigeorgis)
} 
Standard ROA has some connection with the literature on marginal Tobin's $q$ (see, e.g., Abel, 1983; Abel and Eberly, 1994). Following the latter approach, marginal firm value fluctuates along with industry shocks as long as the marginal value stays within a certain range of profitability characterized by lower and upper barriers. When the value process hits one of these boundaries, the firm either invests or divests, so that the marginal value is reflected off the boundaries. We deal here with strategic situations where firms adjust their capital stocks or production capacity in the face of uncertainty and competition (see, e.g., Leahy, 1993; Baldursson, 1998; Baldursson and Karatzas, 1996; Grenadier, 2002). We contribute to the existing literature by highlighting the connection between the theory on marginal Tobin's $q$ under uncertainty and (deterministic) capitalaccumulation games.

In dynamic problems, one often distinguishes several types of controls or strategies. Open-loop or precommitment strategies set ex ante a certain investment path to be pursued regardless of the state realization. In case of closed-loop strategies, the decision makers can condition their actions on the entire state paths. Inbetween, decision makers can employ Markov or feedback strategies, whereby the current state value is considered a sufficient statistic for decision making. This latter type of control is typically employed in standard ROA. Such notions originating from control theory also find application in dynamic game theory, as discussed in Başar and Olsder (1999) and Fudenberg and Tirole (2002). Capital accumulation games — studied in continuous time by e.g., Spence (1979), Fudenberg and Tirole (1983) and Reynolds (1987) — hinge on the distinction and comparison between these different notions of strategies. These models are typically built as differential games that assume a deterministically growing market. Spence (1979) cautions:

"Uncertainty may alter the qualitative conclusions drawn here, even if firms are not riskaverse. Because of the irreversibility, uncertainty acts in part like a further constraint on, or cost of, growth. This aspect of the subject is complicated and in need of further theoretical work as well."

To the best of our knowledge, this research gap has not yet bridged. As noted by Back and Paulsen (2009), models dealing with strategic investment in capital stocks under uncertainty (see 
Leahy, 1993; Baldursson and Karatzas, 1996; Baldursson, 1998) have typically overlooked the inclusion of the capital stock held by rivals in the characterization of the investment strategy, in effect employing strategies that are open-loop with respect to the industry state. ${ }^{1}$ This approach may be ill-advised because Nash equilibria in open-loop strategies often fail to be subgame perfect (see Fudenberg and Tirole, 1991, section 13.4). An emerging strand in the literature turns to closed-loop investment strategies instead. Back and Paulsen (2009) show that the Nash equilibrium obtained in Grenadier (2002) fails subgame perfection and discuss the difficulties in formulating dynamic capacity-expansion problems in closed-loop strategies. Novy-Marx (2009) derives closed-loop equilibrium strategies for duopolistic capacity competition under more restrictive assumptions (than we do). This paper is an attempt to bridge this research gap. We model market uncertainty via Itô processes as standard in ROA and supplement the analysis with techniques from the literature on stochastic differential games. We particularly focus on Markov capital-adjustment strategies and characterize the Markov perfect equilibria. This allows us to quantify the reaction to rivals' strategic capacity adjustment decisions and modify the investment rule on marginal Tobin's $q$ to account for negative strategic externalities on the firm's marginal value.

The paper is organized as follows. Section 2 presents the model assumptions. Section 3 discusses the appropriate choice of strategy space and proposes the use of Markov strategies. Section 4 formulates the problem with Markov controls and derives the Markov perfect equilibria. Section 5 concludes.

\section{Model primitives and assumptions}

Consider a complete filtered probability space $(\Omega, \mathbb{F}, \mathbb{P})$, where the filtration $\mathbb{F} \equiv\left\{\mathscr{F}_{t}\right\}_{t \geq 0}$ is a family of "tribes" that satisfies the usual conditions: $\mathscr{F}_{s} \subseteq \mathscr{F}_{t}$ for all $s<t, \mathscr{F}_{t}=\bigcap_{s>t} \mathscr{F}_{s}$ and the $\mathbb{P}$-null set belongs to $\mathscr{F}_{0}$. The tribe $\mathscr{F}_{t}$ denotes the information available to decision makers at time

$t$. The market is subject to an exogenous shock $X=\left\{X_{t}\right\}_{t \geq 0}$ modeled as a diffusion. The process $X$ starts at $X_{0}=x \in \mathcal{X}$ almost surely, with $\mathcal{X}=\mathbb{R}$ for simplicity; $X$ is the unique (strong) solution

\footnotetext{
${ }^{1}$ An exception is most lump-sum investment models (see, e.g., Smets, 1991; Grenadier, 1996; Huisman and Kort, 1999) that do not suffer from the same weakness as they build on Fudenberg and Tirole's (1985) closed-loop approach.
} 
to the stochastic differential equation (SDE),

$$
\mathrm{d} X_{t}=\mu\left(X_{t}\right) \mathrm{d} t+\sigma\left(X_{t}\right) \mathrm{d} B_{t}
$$

where $B=\left\{B_{t}\right\}_{t \geq 0}$ is a standard Brownian motion. This SDE covers a fairly broad family of stochastic processes used in economic analysis, such as geometric Brownian motion and the OrnsteinUhlenbeck mean-reverting process. To preclude foresight about future economic developments, we assume that $X$ is $\mathbb{F}$-adapted, namely that $X_{t}$ is $\mathscr{F}_{t}$-measurable for all $t \in \mathbb{R}_{+}$. For existence, we also assume that $\left\{\mu\left(X_{t}\right)\right\}_{t \geq 0}$ and $\left\{\sigma\left(X_{t}\right)\right\}_{t \geq 0}$ are $\mathbb{F}$-adapted with finite variations and that $\mu, \sigma: \mathcal{X} \rightarrow \mathbb{R}$ are Lipschitz continuous and satisfy the growth condition.

We consider a symmetric duopoly, where at time $t$ on path $\omega \in \Omega$, firm $i(j)$ has capital stock $K_{t}^{i}(\omega)\left(K_{t}^{j}(\omega)\right){ }^{2}$ Firm $i(j)$ 's initial capital stock is $K_{0-}^{i}=k^{i}\left(K_{0-}^{j}=k^{j}\right)$ almost surely. ${ }^{3}$ The vector of initial industry capital stocks is $k=\left(k^{i}, k^{j}\right)^{\top}$, where $\top$ denotes the transpose. The realization of the capital stocks at time $t$ is given by the two-dimensional vector $K_{t}(\omega)=\left(K_{t}^{i}(\omega), K_{t}^{j}(\omega)\right)^{\top}$.

At any instant $t$, firm $i$ can adjust its capital stock, i.e., acquire new capital or sell unnecessary units. Assume firms face a symmetric, strictly convex capital-adjustment cost function $\kappa: \mathbb{R} \rightarrow \mathbb{R}$, with $\kappa(0)=0$. The function $\kappa(\cdot)$ is differentiable almost everywhere except maybe at $I=0$. An example of such an adjustment cost function is the often-used kinked, piecewise linear cost function,

$$
\kappa(I)=\underline{\kappa} I^{-}+\bar{\kappa} I^{+},
$$

with $(0 \leq) \underline{\kappa}<\bar{\kappa}$ (to preclude arbitrage opportunities) and where, by definition, $I^{+} \triangleq \max \{0, I\}$ and $I^{-} \triangleq \max \{0,-I\}$. Another example is the quadratic investment cost function,

$$
\kappa(I)=a I+\frac{b}{2} I^{2}
$$

with $a, b \in \mathbb{R}_{+}$. We denote the (partial) derivative of function $f$ with respect to argument $y$ by $f_{y}(\cdot) . \kappa_{I}(\cdot)$ is the marginal investment cost. Denote the left (resp., right) limit of $\kappa_{I}(\cdot)$ by $\underline{\kappa}_{I}$ (resp.,

\footnotetext{
${ }^{2}$ To reduce mathematical complexity, we here consider for each firm a one-dimensional capital stock. Considering multiple resources would require to examine whether the resources are complements or substitutes in addition to the factors analyzed herein.

${ }^{3}$ We distinguish between time $0-$ and 0 because a lump-sum investment may occur right at the outset, immediately adjusting the capital stock held.
} 
$\bar{\kappa}_{I}$ ). Function (3) is differentiable at $I=0$, while function (2) is not, having left-limit $\underline{\kappa}_{I}=\underline{\kappa}$ and right-limit $\bar{\kappa}_{I}=\bar{\kappa}$.

Following Baldursson (1998), an investment strategy is a capital-accumulation process $\xi^{i}$ with decomposition $\xi^{i} \triangleq \xi^{i+}-\xi^{i-}$, where the negative and the positive parts of the process $-\xi^{i-}$ and $\xi^{i+}$, respectively — are right-continuous with left-limits, nondecreasing, and $\mathbb{F}$-adapted. Besides, $\xi^{i+}(0-)=\xi^{i-}(0-)=0$ almost surely. The process $\xi^{i}$ has (pathwise) bounded variation as the difference between two nondecreasing processes. Following investment strategy $\xi^{i}$, firm $i$ 's capital stock at time $t$ on path $\omega \in \Omega$ is

$$
K_{t}^{i}(\omega)=k^{i}+\xi_{t}^{i+}(\omega)-\xi_{t}^{i-}(\omega)
$$

An instantaneous change in capital stock is denoted $\mathrm{d} \xi_{t}(\omega)=\xi_{t}^{i}(\omega)-\xi_{t-}^{i}(\omega)$. The strategy choice has no impact on the exogenous state dynamics given in equation (1). If it were possible to determine an "investment rate," we could define - as in capital-accumulation (differential) games - a control policy prescribing for firm $i$ an investment rate $I_{t}^{i}$ at time $t \in \mathbb{R}_{+}{ }^{4}$ An instantaneous change at time $t$ in firm $i$ 's capital stock is then $I_{t}^{i}$, with the capital stock evolving according to the ordinary differential equation:

$$
\dot{K}_{t}^{i}=I_{t}^{i}
$$

The industry-wide shock $X=\left\{X_{t}\right\}_{t \geq 0}$ follows the Itô process of equation (1), while the capital stocks evolve according to equation (4). At each instant $t$, firm $i$ receives an unknown (gross) profit flow $\pi^{i}\left(X_{t}(\omega), K_{t}(\omega)\right)$ depending on the shock realization $X_{t}(\omega)$ and the prevalent capital stocks of the rivals, $K_{t}^{i}(\omega)$ and $K_{t}^{j}(\omega)$. The profit function $\pi^{i}$ is strictly increasing and twice continuously differentiable in $x$; it is continuously differentiable, strictly decreasing in $k^{j}$, and concave in $k^{i}$. We assume that the marginal profit is increasing in the shock $\left(\pi_{k^{i} x}^{i}>0\right)$; this ensures that the optimal strategy can be expressed in terms of divestment/ investment triggers. ${ }^{5}$ Also assume that the profit function is submodular, with $\pi_{k^{i} k^{j}}<0$. Although not necessary, we can

\footnotetext{
${ }^{4} \mathrm{~d} \xi_{t}(\omega)$ is a Lebesgue-Stieltjes differential and cannot properly be interpreted in terms of investment rate since the capital stocks evolve over time but in a "singular" manner.

${ }^{5}$ We also impose Inada conditions on the profit function: $\lim _{k^{i} \rightarrow 0} \pi_{k^{i}}^{i}(x, k)=\infty$ and $\lim _{k^{i} \rightarrow \infty} \pi_{k^{i}}^{i}(x, k)=0$.
} 
specialize the reduced-form profit function. Consider, for instance, that the price $P_{t}(\omega)$ that clears the market is given by $P_{t}(\omega)=D\left(X_{t}(\omega), K_{t}^{i}(\omega)+K_{t}^{j}(\omega)\right)$, where the demand function $D(\cdot, \cdot)$ is strictly increasing and twice continuously differentiable in $x$ and strictly decreasing in $k^{i}$ and $k^{j}$. For negligible production cost, firm $i$ 's instantaneous net profit is $k^{i} D\left(x, k^{i}+k^{j}\right)-\kappa(I)$, which is increasing and twice continuously differentiable in $x$.

Firms are assumed risk-neutral, facing a common, constant risk-free rate $r{ }^{6}$ The payoff to firm $i$ if the strategy profile $\left(\xi^{i}, \xi^{j}\right)$ is followed is

$$
J^{i}\left(x, k \mid \xi^{i}, \xi^{j}\right) \triangleq \mathbb{E}\left[\int_{0}^{\infty} e^{-r t} \pi\left(X_{t}, K_{t}\right) \mathrm{d} t-\int_{0}^{\infty} e^{-r t} \kappa\left(\mathrm{d} \xi_{t}^{i}\right) \mid x, k\right]
$$

where the second integral is understood (pathwise) as a Lebesgue-Stieljes integral. The firms adjust their capital stock with a view to maximizing their expected payoffs. If a Nash equilibrium $\xi^{*}=$ $\left(\xi^{i *}, \xi^{j *}\right)$ exists, the value received by firm $i$ in state $(x, k)$ is

$$
V^{i}(x, k) \triangleq \max _{\xi^{i}} J^{i}\left(x, k \mid \xi^{i}, \xi^{j *}\right)
$$

Given the model assumptions, $V^{i}$ is strictly increasing, twice continuously differentiable in the shock. Besides, $V_{x k^{i}}^{i}>0$ and $V_{k^{i} k^{j}}^{i}<0$.

In the above definition of strategy space, it is not clear how the arrival of new information about the rival's realized capital stock, namely the information structure, influences the capital adjustment strategy. This modeling choice is, however, essential to characterize the equilibrium outcomes, as discussed below.

\section{Strategy-space choice and solution approach}

In dynamic problems, the choice of the strategy space is closely related to the hypothesized information structure. Two information structures are common in the deterministic control and differential games literature. Open-loop or precommitment strategies prescribe a certain investment rate at each time $t$ of the control horizon. Markov strategies enable the decision maker to condition

\footnotetext{
${ }^{6}$ The assumption of risk neutrality can be relaxed if arbitrage opportunities in the market do not exist and the market is complete (see Cox and Ross, 1976; Harrison and Kreps, 1979). Introducing capital depreciation in the model would not add much flavor; it only increases the discount factor by the depreciation rate.
} 
her decision on the latest value of the state. In deterministic single-agent control problems, this distinction is not material since these assumptions lead to different representations of the same optimal policy (see Başar and Olsder, 1999, section 5.6). When competition or uncertainty are involved, the different types of strategies may lead to different equilibrium outcomes. The definition of $\xi^{i}$ above is not precise enough to describe the hypothesized information set of player $i$ at each time $t$. A crucial but non-trivial element in defining the strategy space relates to the behavioral conjectures on how firm $j$ 's capital stock feeds back into firm $i$ 's investment or divestment choices at each time over the game play. Fudenberg and Tirole (1985) face a related problem in a deterministic investment investment timing game: a player's mixed strategy defined by a nondecreasing, rightcontinuous, $[0,1]$-valued function (interpreted as the cumulative probability of investing) is not rich enough to accomodate consideration of dynamic strategic interactions over the play of the game. One should thus consider as part of a player's strategy an additional "atoms function" that prescribes a certain intensity of investment at each time $t$ (on path $\omega \in \Omega$ in the stochastic case).

The literature on incremental capital adjustment under uncertainty and competition oftentimes fails to explicitly address this point, relying in effect on the notion of a "myopic firm." A myopic firm essentially assumes that there will be no further changes in the industry capital stock other than those arising from its own investment or divestment decisions. Its value is $J^{i}\left(x, k \mid \xi^{i}, 0\right)$. Myopic strategies are Markovian in the exogenous state $x$ but not in rival firm $j$ 's capital stock. If a firm invests according to such a strategy, it either cannot observe the rivals' investment behavior or it chooses to precommit to a myopic investment path as a function of the actual exogenous state realization.

Grenadier (2002, proposition 3) and Back and Paulsen (2009, equation 8) appear to make an attempt to circumvent the notion of the myopic firm. There, firm $i$ conjectures that, on the equilibrium path, the rival's capital stock is a deterministic function of firm $i$ 's capital stock, $K_{t}^{j}(\omega)=h\left(K_{t}^{i}(\omega)\right)$. In the $n$-firm case, they explicitly consider that frim $i$ 's rivals hold a capital stock of $(n-1) K_{t}^{i}(\omega)$ together. Then, what seems to be an investment strategy which depends on the realization of rival's capital stock is actually an investment strategy which depends on firm $i$ 's capital stock, $K_{t}^{i}(\omega)$, solely. 
In other words, this firm assumes a parallel development of rival's capital stock with no possibility for the rival to deviate from the capital-stock development path conjectured by the myopic firm. This statement is not a derived equilibrium outcome but simply a modeling assumption. Given this behavorial assumption on firm $i$, the Nash equilibrium in which firms follow the latter type of strategy turns out to correspond to the "myopic equilibrium" whereby firm $i$ explicitly ignores rival's realized capital-stock adjustments (Grenadier, 2002, section 2). The above investment strategies are Markovian in the exogenous state but fail to allow the rivals' actual capital stock to feed back into the firm's strategy. The literature on (stochastic) differential games provides mechanisms to allow states driven by the actions of rivals to influence a firm's own decisions. In the next section, we employ such methods.

\section{Markov perfect equilibrium}

Consider that all states are perfectly observable, including the level of capital stock held by the rival firm. Following a Markov strategy, the capital-adjustment decision is made in view of the state realization $(x, k)$. In this case, the history of the game matters only through the present consequences of all past decisions, as reflected in the state vector $(x, k)$. The Nash equilibrium in Markov strategies is a Markov Nash Equilibrium. ${ }^{7}$ A Markov Nash equilibrium is perfect if it is a Markov Nash equilibrium for all state vectors $\left(x, k^{i}, k^{j}\right)$, even those off the equilibrium path. Formally, a Markov Nash equilibrium profile is a Markov perfect equilibrium (MPE) if, for all states $\left(x, k^{i}, k^{j}\right)$, it holds that

$$
V^{i}(x, k)=\max _{\xi_{i}} J^{i}\left(x, k \mid \xi^{i}, \xi^{j *}\right)
$$

Setting arbitrarily $k^{j}=h\left(k^{i}\right)$ fails to ensure a Nash equilibrium for values of $k^{j}$ off the path $h\left(k^{i}\right)$. Introducing an additional term - $\phi$, here — makes it possible to account in firm $i$ 's strategy for the rival's capacity adjustments through the dependence on the states $x, k^{i}$, and $k^{j}$. The investment

\footnotetext{
${ }^{7}$ Because both the payoffs and the strategies depend on the current state realization, we call this a "state-space" game. Dynamic games of complete information are generally solved by means of the perfect Nash equilibrium solution concept. We adapt the usual definition of perfection to a state-space game by identifying information sets in the extensive-form game with points in the state space.
} 
intensity $\xi_{t}^{i}-\xi_{t-}^{i} \neq 0$ is given by $\phi^{i}\left(X_{t}, K_{t}\right) .{ }^{8}$ We next derive sufficient conditions for existence of a Markov perfect equilibrium.

Proposition 1. Firm $i$ 's value in state $(x, k), V^{i}(x, k)$, is the solution of the HJB equation

$$
r V^{i}(x, k)=\max _{I \in \mathbb{R}}\left\{\pi^{i}(x, k)-\kappa(I)+V_{k^{i}}^{i}(x, k) I+V_{k^{j}}^{i}(x, k) \phi^{j}(x, k)+\mathscr{D}_{x} V^{i}(x, k)\right\}
$$

where $\mathscr{D}_{x} V^{i}(x, k)$ indicates an instantaneous value change for an infinitesimal change in the shock, with the operator $\mathscr{D}_{x}$ given by

$$
\mathscr{D}_{x}=\mu(x) \frac{\partial}{\partial x}+\frac{1}{2} \sigma(x)^{2} \frac{\partial^{2}}{\partial x^{2}} .
$$

Proof. Bellman's principle of optimality builds upon the recursive nature of the payoff function in equation (6). For a short time interval of length $t$, the continuation payoff of firm $i$ if the rival follows strategy $\xi^{i *}$ is

$$
\begin{aligned}
J^{i}\left(x, k \mid \xi^{i}, \xi^{j *}\right) & \approx\left\{\pi^{i}(x, k)-\kappa(I)\right\} t+\mathbb{E}\left[\mathbb{E}\left[\int_{t}^{\infty}\left\{\pi^{i}\left(X_{s}, K_{s}\right)-\kappa(I)\right\} \mathrm{d} s \mid X_{t}(\omega), K_{t}(\omega)\right] \mid x, k\right] \\
& \approx\left\{\pi^{i}(x, k)-\kappa(I)\right\} t+\mathbb{E}\left[J^{i}\left(X_{t}(\omega), K_{t}(\omega) \mid \xi^{i}, \xi^{j *}\right) \mid x, k\right]
\end{aligned}
$$

By taking the supremum,

$$
\begin{aligned}
V^{i}(x, k) & \approx \max _{I \in \mathbb{R}}\left\{\left[\pi^{i}(x, k)-\kappa(I)\right] t+\max _{\xi^{i}} \mathbb{E}\left[J^{i}\left(X_{t}(\omega), K_{t}(\omega) \mid \xi^{i}, \xi^{j *}\right) \mid x, k\right]\right\} \\
& \approx \max _{I \in \mathbb{R}}\left\{\left[\pi^{i}(x, k)-\kappa(I)\right] t+\mathbb{E}\left[V^{i}\left(X_{t}(\omega), K_{t}(\omega)\right) \mid x, k\right]\right\}
\end{aligned}
$$

Substract $V^{i}(x, k)$ from both sides, divide by $t(>0)$ and take the limit as $t \rightarrow 0$. Equation (8) then obtains, whereby $V^{i}$ is presumed twice continuously differentiable in $x$ and once continuously differentiable in $k^{i}$ and $k^{j}$, with

$$
\lim _{t \downarrow 0} \frac{\mathbb{E}\left[V^{i}\left(X_{t}(\omega), K_{t}(\omega)\right) \mid x, k\right]-V^{i}(x, k)}{t}=V_{k^{i}}^{i}(x, k) I+V_{k^{j}}^{i}(x, k) \phi^{j}(x, k)+\mathscr{D}_{x} V^{i}(x, k) .
$$

It is desirable to give more intuition (via the stochastic maximum principle) concerning the interpretation of HJB equation (8) and further characterize the induced optimal behavior. Consider a vector of adjoint variables for firm $i, \lambda^{i}=\left(\lambda^{i i}, \lambda^{i j}\right)^{\top}$. Define the deterministic Hamiltonian

$$
H^{i}\left(x, k, I^{i}, I^{j}, \lambda^{i}\right) \triangleq \pi^{i}(x, k)-\kappa\left(I^{i}\right)+\lambda^{i i} I^{i}+\lambda^{i j} I^{j}
$$

Using the notation of equation (9) in HJB equation (8), player $i$ 's value function $V^{i}$ solves for each $(x, t) \in \mathcal{X} \times \mathbb{R}_{+}$the HJB equation

$$
r V^{i}(x, k)=\max _{I \in \mathbb{R}}\left\{H^{i}\left(x, k, I, \phi^{j}(x, k), V_{k}^{i}(x, k)\right)+\mathscr{D}_{x} V^{i}(x, k)\right\}
$$

\footnotetext{
${ }^{8}$ Although it is not mathematically rigorous to define investment rate in the present setting, we can nevertheless use the heuristics in Abel and Eberly (1994): the change in capital stock is interpreted as analogous to an investment rate $I$, allowing the derivation of a Hamilton-Jacobi-Bellman (HJB) equation.
} 
where $V_{k}^{i}=\left(V_{k^{i}}^{i}, V_{k^{j}}^{i}\right)^{\top}$ is the gradient of firm $i$ 's value function w.r.t. the vector of capital stocks. In other words, the HJB equation (8) can be understood as a static optimization problem, whereby firm $i$ is not only looking at maximizing its current net profit flow $\pi^{i}(x, k)-\kappa\left(I^{i}\right)$ but considers also the future, indirect marginal value contribution of its current investment via the shadow price vector $\lambda^{i}=V_{k}^{i}$. Here, the rival's reaction to changing industry capital stock $k^{i} \mapsto \phi^{j}(x, k)$ with $k=\left(k^{i}, k^{j}\right)^{\top}$ is explicitly considered when determining the optimal investment intensity $\phi^{i}(x, k) \in \mathbb{R}$. Player $i$ cares about how his opponent will react to a change in its own capital stock, with the indirect marginal value contribution being $\phi^{i}(x, k) V_{k^{j}}^{i}(x, k)$. A pair $\left(\phi^{i}(x, k), \phi^{j}(x, k)\right)$ forms a Markov Nash equilibrium in the static, continuous two-player game if firm values satisfy equation (10). To obtain a Markov perfect equilibrium (MPE), we must be able at each $(x, k)$ to find such a pair $\left(\phi^{i}(x, k), \phi^{j}(x, k)\right)$.

Owing to the second-order term in the HJB equation, it is difficult (even in one-player settings) to find closed-form solutions for the value functions $V^{i}$, unless certain "smoothness" restrictions are imposed. In the present stochastic case with two players, a system of coupled second-order partial differential equations obtains, making the derivation of a closed-form solution even more challenging. Nevertheless, we may characterize the equilibrium further even if closed-form solutions are not readily determinable. Denote the marginal value of firm $i$ to a change in its own capital stock by $v^{i}(x, k) \triangleq V_{k^{i}}^{i}(x, k)$. At places, we drop the dependence of the shadow price $v^{i}$ on the state $(x, k)$ when no confusion may arise. By isolating terms depending on $I$ in HJB equation (8), the maximum condition simplifies to the static optimization problem

$$
f\left(v^{i}\right) \triangleq \max _{I \in \mathbb{R}}\left\{v^{i} I-\kappa(I)\right\} \quad \text { or } \quad \phi^{i}(x, k)=\arg \max _{I \in \mathbb{R}}\left\{v^{i} I-\kappa(I)\right\} .
$$

The objective function in equation (11) is strictly concave in $I$ and $\kappa(\cdot)$ is differentiable everywhere on $(-\infty, 0)$ and $(0, \infty)$. In these regions, a set of first-order conditions is sufficient to determine the optimal investment rule. When firm $i$ invests or divests $\left(\phi^{i}(x, k) \neq 0\right)$, the MNE strategy in state $(x, k)$ prescribes that

$$
v^{i}=\left(\kappa_{I} \circ \phi^{i}\right)(x, k)
$$

with the symbol "o" indicating coumpound functions. In other words, when firm $i$ divests, the MNE 
strategy prescribes to invest up to the level where the marginal cost of adding capital, $\left(\kappa_{I} \circ \phi^{i}\right)(x, k)$, equals the marginal value of capital, $v^{i}(x, k)$. A similar result holds for the divestment case. This investment policy is analogous to the theory prescription on marginal Tobin's $q$ with the additional consideration of competition in the market. By the chain rule, it obtains that

$$
v_{x}^{i}=\left(\kappa_{I I} \circ \phi^{i}\right)(x, k) \times \phi_{x}^{i}(x, k) .
$$

For a sufficiently smooth function $V^{i}$, we have $V_{x k^{i}}^{i}=V_{k^{i} x}^{i}$, with $V_{x k^{i}}^{i}>0$ (by assumption). Since $\kappa(\cdot)$ is strictly convex $\left(\kappa_{I I}>0\right)$ and $v_{x}^{i}>0$, it obtains that $\phi_{x}^{i}>0$ or firm $i$ invests more for improved economic conditions or vice versa, all other things remaining equal. We readily note an unexpected aspect of competition on the firms' reactions to economic shocks. Define

$$
R^{i}\left(x, k, I^{j}\right) \triangleq \arg \max _{I \in \mathbb{R}}\left\{H^{i}\left(x, k, I, I^{j}, V_{k}^{i}(x, k)\right)+\mathscr{D}_{x} V^{i}(x, k)\right\}
$$

$R^{i}(x, k, \cdot)$ is firm $i$ 's reaction function to firm $j$ 's investment intensity. In the Nash equilibrium of the static game, $\phi^{i}(x, k)=R^{i}\left(x, k, \phi^{j}(x, k)\right)$. We wish to capture the influence of the shock on the investment intensity of firm $i$. By total differentiation,

$$
\frac{\mathrm{d} \phi^{i}}{\mathrm{~d} x}(x, k)=\frac{\partial R^{i}}{\partial x}+\frac{\partial R^{i}}{\partial I^{j}} \frac{\partial \phi^{j}}{\partial x}(x, k)
$$

The second right-hand term captures the strategic effect in reaction to a shock. Since $\frac{\partial R^{i}}{\partial I^{j}}<0$ (strategic substitute) and $\frac{\partial \phi^{j}}{\partial x}(x, k)>0$, the intensity of investment in state $(x, k)$ is decreased for firm $i$ compared to the myopic situation $\left(\partial R^{i} / \partial x\right)$. In other words, the intensity with which a firm increases (resp., decreases) its capital stock in response to favorable (resp., unfavorable) industry shocks is lower under competition. This effect, arising under competition, creates a natural hedge for the firm with less erratic changes in capital stocks. By applying the chain rule to equation (12), it obtains

$$
\begin{aligned}
& v_{k^{i}}^{i}(x, k)=\left(\kappa_{I I} \circ \phi^{i}\right)(x, k) \times \phi_{k^{i}}^{i}(x, k), \\
& v_{k^{j}}^{i}(x, k)=\left(\kappa_{I I} \circ \phi^{i}\right)(x, k) \times \phi_{k^{j}}^{i}(x, k) .
\end{aligned}
$$

Since $V^{i}$ is concave in $k^{i}, \phi_{k^{i}}^{i}<0$ follows from equation (13a), so the firm is less responsive to a favorable shock when it already has a large capital stock. By submodularity of the profit function 
$\left(\pi_{k^{i} k^{j}}^{i}<0\right)$, we have $v_{k^{j}}^{i}<0$. Thus from equation (13b), $\phi_{k^{j}}^{i}<0$ or firm $i$ invests less when its rival has a large capital stock $k^{j}$. The firm with a headstart (larger capital stock) thus can discipline the smaller firm to refrain from investing. In other words, if a firm increases its capital stock, rivals react to an increased industry capital stock by reducing their own capital stocks. This preemptive effect is not captured when one considers myopic strategies.

It is suitable to partition the state space to determine which action to take in each state $(x, k)$. Since $\kappa(\cdot)$ is strictly convex, $\kappa_{I}(I)<\underline{\kappa}_{I}$ for all $I \in(-\infty, 0)$ and $\bar{\kappa}_{I}<\kappa_{I}(I)$ for all $I \in(0, \infty)$. From equation $(12)$, the regions $(x, k)$ where divestment or investment occurs are therefore such that $v^{i}(x, k) \in\left(-\infty, \underline{\kappa}_{I}\right]$ and $v^{i}(x, k) \in\left[\bar{\kappa}_{I}, \infty\right)$, respectively. The optimal investment rule prescribes not to invest or $\phi\left(x, k^{i}\right)=0$ if $v^{i}(x, k) \in\left(\underline{\kappa}_{I}, \bar{\kappa}_{I}\right)$. The investment policy thus reads:

$$
\phi^{i}(x, k)=\left\{\begin{array}{llll}
<0 & \text { if } & v^{i}(x, k) \in\left(-\infty, \underline{\kappa}_{I}\right], \\
=0 & \text { if } & v^{i}(x, k) \in\left(\underline{\kappa}_{I}, \bar{\kappa}_{I}\right), \\
>0 & \text { if } & v^{i}(x, k) \in\left[\bar{\kappa}_{I}, \infty\right) .
\end{array}\right.
$$

If $\kappa(\cdot)$ is differentiable at $I=0$ as in the quadratic case of equation (3), then $\underline{\kappa}_{I}=\bar{\kappa}_{I}$ and $\left(\underline{\kappa}_{I}, \bar{\kappa}_{I}\right)$ is empty. If $\kappa(\cdot)$ is not differentiable at $I=0$ - as in the case of the kinked, linear adjustment cost function in equation $(2)$ - with $\underline{\kappa}_{I}<\bar{\kappa}_{I}$ (by strict convexity of the cost function), $\left(\underline{\kappa}_{I}, \bar{\kappa}_{I}\right)$ is nonempty.

Following the marginal Tobin's $q$ approach, the investment policy characterized in equation (14) is driven by the marginal value from investing and the marginal cost incurred by the firm upon investing. The firm "sells" capital (divests) to prevent the marginal revenue product of capital $v^{i}$ from falling below a certain lower profitability level $\underline{\kappa}_{I}$ and acquires capital (invests) to prevent $v^{i}$ from rising above an upper profitability level $\bar{\kappa}_{I}$. If the open interval between these thresholds $\left(\underline{\kappa}_{I}, \bar{\kappa}_{I}\right)$ is nonempty - as in the case of equation $(2)$ - the set of states $(x, k)$ whereby $v^{i}(x, k) \in\left(\underline{\kappa}_{I}, \bar{\kappa}_{I}\right)$ is an inaction or hysteresis region where the firm neither invests nor divests ("stays put" or waits). Within the bands $\underline{\kappa}_{I}$ and $\bar{\kappa}_{I}$, the shadow price process is driven solely by the exogenous shock (i.e., no control is exercised). At the upper barrier $\bar{\kappa}_{I}$, firms will be indifferent between staying put or expanding their capital stock, while at the lower barrier $\underline{\kappa}_{I}$, firms will be indifferent between holding 
the current level of capital stock constant or downsizing. Expansion at $\bar{\kappa}_{I}$ and downsizing at $\underline{\kappa}_{I}$ maximize the present discounted value of firm $i$ 's profits in Markov perfect equilibrium. Given the nature of the problem (singular control), the marginal value is always located inside the hysteresis (wait) region. ${ }^{9}$

Consider next the dynamics of the shadow price. Differentiating HJB equation (8) w.r.t. $k_{i}$ yields (for sufficiently smooth function)

$$
r v^{i}(x, k)=\pi_{k^{i}}^{i}(x, k)+v_{k^{i}}^{i}(x, k) \phi^{i}(x, k)+V_{k^{j}}^{i}(x, k) \phi_{k^{i}}^{j}(x, k)+v_{k^{j}}^{i}(x, k) \phi^{j}(x, k)+\mathscr{D}_{x} v(x, k) .
$$

The left-hand side is the required return on the value of a marginal unit of capital. The right-hand side of equation (15) is the expected return; it consists of the marginal operating profit, $\pi_{k^{i}}^{i}(x, k)$, the indirect value contributions, and the marginal expected gain $\mathscr{D}_{x} v(x, k)$. Equation (15) is thus a no-arbitrage condition to be satisfied at each time and in each state $(x, k)$ in Markov perfect equilibrium. If a strategy $\phi^{i}(x, k)$ solves the maximization problem of equation (15), the equilibrium shadow price process obtains as the solution of a second-order partial differential equation. The term $V_{k^{j}}^{i}(x, k) \phi_{k^{i}}^{j}(x, k)$ is the strategic effect term: it captures the impact on firm $i$ 's value from the reaction (capital stock change) by firm $j$. Since $\phi_{k^{i}}^{j}<0$, this term constitutes a negative externality on the marginal value of capital. Because of the cross influence, the evolution of the shadow price $v^{i}$ is determined by a system of coupled partial differential equations.

Consider now how the policy influences the dynamic behavior of the shadow price process. After a possible initial discrete adjustment at time 0 (indeed at time $0-$ ) occurring if the initial state $(x, k)$ is such that $v^{i}(x, k) \notin\left(\underline{\kappa}_{i}, \bar{\kappa}_{I}\right)$, the optimal policy of firm $i$ involves adjusting the capital stock process $K^{i}$ at the lower and upper thresholds so the firm sustains a certain level (region) of profitability $\left(\underline{\kappa}_{I}, \bar{\kappa}_{I}\right)$. The shadow price evolution thus admits a lower and upper reflecting barrier, whereby the shadow price evolves according to the partial differential equation (15) within the hysteresis region.

\footnotetext{
${ }^{9}$ The set of times at which investment or divestment occurs has Lebesgue measure zero, the marginal value process being located within the bands almost surely. If $v^{i}\left(X_{t}, K_{t-}\right)$ were outside this region, an instantaneous control of sufficiently large intensity $\xi_{t}^{i}-\xi_{t-}^{i}$ would adjust the capital stock up or down to ensure that $v^{i}\left(X_{t}, K_{t}\right)$ is located in $\left(\underline{\kappa}_{I}, \bar{\kappa}_{I}\right)$.
} 


\section{Conclusion}

The present modeling approach is useful for analyzing dynamic capacity investment decisions in industries subject to stochastic growth and competitive dynamics. We adopted a fairly general approach that covers a large family of stochastic processes used in economic analysis and many reduced-form profit functions. The characterization of the appropriate strategy space is critical to determining equilibria in real option models that involve competition under uncertainty. When firms can observe and react to rivals' actions over time, the equilibrium solution should form a Markov perfect equilibrium. However, deriving the Markov Perfect Equilibrium is usually more involved than deriving the myopic equilibrium since an additional partial derivative linked to a strategic effect obtains in the HJB equation.

In the presence of a convex capital-adjustment cost, the investment rule on marginal Tobin's $q$ still holds. If the investment is costlessly reversible (differentiability at $I=0$ ), the model produces the classic result on marginal Tobin's $q$ : at each date (in each state) the capital stock $K^{i}$ is adjusted to equate the (ever-changing) marginal revenue product of capital $v^{i}(x, k)$ with marginal cost of capital. If the investment costs are partly unrecoupable (non-differentiability) then there exists a hysteresis region in which the firm waits to invest or divest. In Markov perfect equilibrium, the intensity of investment with which a firm invests is affected by the presence of rivals. The firm with the larger capital stock disciplines its smaller rival away from investing. Besides, the presence of competition creates a hedging effect: under competition, a firm invests with less intensity than if it were ignoring competition.

\section{References}

Abel, A. B. (1983). Optimal investment under uncertainty. American Economic Review 73(1), $228-233$.

Abel, A. B. and J. C. Eberly (1994). A unified model of investment under uncertainty. American Economic Review 84(5), 1369-1384. 
Back, K. and D. Paulsen (2009). Open-loop equilibria and perfect competition in option exercise games. Review of Financial Studies 22(11), 4531-4552.

Başar, T. and G. J. Olsder (1999). Dynamic Noncooperative Game Theory (2 ed.). Society for Industrial and Applied Mathematics.

Baldursson, F. M. (1998). Irreversible investment under uncertainty in oligopoly. Journal of Economic Dynamics and Control 22, 627-644.

Baldursson, F. M. and I. Karatzas (1996). Irreversible investment and industry equilibrium. Finance and Stochastics 1(1), 69-89.

Chevalier-Roignant, B., C. M. Flath, A. Huchzermeier, and L. Trigeorgis (2010). Strategic investment under uncertainty: a synthesis. SSRN working papers.

Chevalier-Roignant, B. and L. Trigeorgis (2011). Competitive Strategy: Options and Games. The MIT Press, forthcoming.

Cox, J. C. and S. A. Ross (1976). The valuation of options for alternative stochastic processes. Journal of Financial Economics 3(1-2), 145-166.

Dixit, A. K. and R. S. Pindyck (1994). Investment under Uncertainty. Princeton University Press.

Fudenberg, D. and J. Tirole (1983). Capital as commitment: Strategic investment to deter mobility. Journal of Economic Theory 250, 227-250.

Fudenberg, D. and J. Tirole (1985). Preemption and rent equilization in the adoption of new technology. Review of Economic Studies 52(3), 383-401.

Fudenberg, D. and J. Tirole (1991). Game Theory. The MIT Press.

Fudenberg, D. and J. Tirole (2002). Dynamic models of oligopoly. Harwood Fundamentals of Applied Economics, Routledge.

Grenadier, S. R. (1996). The strategic exercise of options: Development cascades and overbuilding in real estate markets. Journal of Finance 51(5), 1653-1679. 
Grenadier, S. R. (2002). Option exercise games: An application to the equilibrium investment strategies of firms. Review of Financial Studies 15(3), 691-721.

Harrison, J. M. and D. Kreps (1979). Martingales and arbitrage in multiperiod securities markets. Journal of Economic Theory 20(3), 381-408.

Huisman, K. J. M. and P. M. Kort (1999). Effects of strategic interactions on the option value of waiting. Working paper, Tilburg University.

Leahy, J. V. (1993). Investment in competitive equilibrium: The optimality of myopic behavior. Quarterly Journal of Economics 108(4), 1105-1133.

Novy-Marx, R. (2009). Preempting Preemptive Investment. Working paper, University of Chicago.

Reynolds, S. S. (1987). Capacity investment, preemption and commitment in an infinite horizon model. International Economic Review 28(1), 69-88.

Smets, F. (1991). Exporting Versus Foreign Direct Investment: The Effect of Uncertainty, Irreversibilities and Strategic Interaction. Ph. D. thesis, Yale University, New Haven, CT.

Spence, A. M. (1979). Investment strategy and growth in a new market. Bell Journal of Economics 10(1), 1-19.

Trigeorgis, L. (1996). Real Options: Managerial Flexibility and Strategy in Resource Allocation. The MIT Press. 